Bergner M, Bobbitt RA, Kressel S, Pollard WE, Gilson BS, Morris JR. The sickness impact profile: conceptual formulation and methodology for the development of a health status measure. Int J Health Serv 1976;6:393-415.

4 Hunt SM, McKenna SP, McEwen J, Backett EM, Williams J, Papp E. A quantitative approach to perceived health status: a validation study. J Epidemiol Community Health 1980;34:281-6.

5 Torrance GW, Furlong W, Feeny D, Boyle M. Multi-attribute preference functions. Pharmacoeconomics 1995;7:503-20.

6 Ware JE, Sherbourne CD. The MOS 36-item short-form health survey (SF-36). I. Conceptual framework and item selection. Med Care 1992;30:473-83.

7 Sintonen $\mathrm{H}$. An approach to measuring and valuing health states. Soc Sci Med 1981;15:55-65.

EuroQoL Group. EuroQoL-a new facility for the measurement of health-related quality of life. Health Policy 1990;16:199-208.

9 Brooks RG. EuroQoL-the current state of play. Health Policy 1996;37:5372.

10 Williams AH. The measurement and valuation of health: a chromicle. University of York: Centre for Health Economics, 1995. (Discussion paper 136.)

11 Brazier J, Jones N, Kind P. Testing the validity of the EuroQoL and comparing it with the SF-36 health survey questionnaire. Qual Life Res 1993;2:169-80

12 Van Agt H, Essink-Bot M-L, Krabbe P, Bonsel G. Test-retest reliability of health state valuations collected with the EuroQoL questionnaire. Soc Sci Med 1994:39:1537-44

13 Essink-Bot M-L, Krabbe P, Bonsel G, Aaronson N. An empirical comparison of four generic health status measures: the Nottingham health profile, the medical outcomes study 36 -item short-form health survey, the COOP/WONCA charts, and the EuroQoL Instrument. Med Care 1997;35:522-37.

14 Hurst NP, Jobanputra P, Hunter M, Lambert M, Lochead A, Brown H. Validity of EuroOoL - a generic health status instrument-in patients with rheumatoid arthritis. Br J Rheumatol 1994;33:655-62.
15 Sculpher M, Dwyer N, Byford S, Stirrat G. Randomised trial comparing hysterectomy and transcervical endometrial resection: effect on health related quality of life and costs two years after surgery. Br J Obstet Gynaecol related quality of lif

16 Hollingworth W, Mackenzie R, Todd CJ, Dixon AK. Measuring changes in quality of life following magnetic resonance imaging of the knee: SF-36, EuroQoL or Rosser index? Qual Life Res 1995;4:325-34.

17 Erens B. Health-related quality of life: general population survey. London: Social and Community Planning Research, 1994. (Technical report.)

18 Kish L. Survey sampling. New York: Wiley, 1965.

19 Thomas M, Goddard E, Hickman M, Hunter P. The general household survey 1992. London: HMSO, 1994. (OPCS Series GHS No 23.)

20 Martin J, Letzer H, Elliot D. The prevalence of disability among adults. OPCS surveys of disability in Great Britain. Report 1. London: HMSO, 1988.

21 Dolan P, Gudex C, Kind P, Williams A. A social tariff for EuroOoL: results from a UK general population survey. University of York: Centre for Health Economics, 1995. (Discussion paper 138.)

22 Department of Health and Social Security. Prevention and health: everybody's business. A reassessment of public and personal health. London: HMSO, 1976

23 Black D, Morris JN, Smith C, Townsend P. Black Report. Inequalities in health: report of a research working group. London: Department of Health and Social Security, 1980.

24 Rahkonen O, Arber S, Lahelma E. Health inequalities in early adulthood: a comparison of young men and women in Britain and Finland. Soc Sci Med 1995;41:163-71.

25 Prescott-Clarke P, Primatesta P, eds. Health survey for England, 1996. London: Stationery Office, 1998.

26 Weinstein MC, Siegel JE, Gold MR, Kamlet MS, Russell LB. Recommendations of the panel on cost-effectiveness in health and medicine. JAMA 1996;276:1253-8.

(Accepted 31 October 1997)

\title{
Use of calcium channel blockers and risk of suicide: ecological findings confirmed in population based cohort study
}

\author{
Gunnar Lindberg, Kerstin Bingefors, Jonas Ranstam, Lennart Råstam, Arne Melander
}

\begin{abstract}
Objective: To investigate possible associations between use of cardiovascular drugs and suicide. Design: Cross sectional ecological study based on rates of use of eight cardiovascular drug groups by outpatients. A population based cohort study including users of drugs to control hypertension. Subjects: The ecological study included 152 of Sweden's 284 municipalities. The cohort study included all inhabitants of one Swedish municipality who during 1988 or 1989 had purchased cardiovascular agents from pharmacies within the municipality. Six hundred and seventeen subjects $(18.2 \%)$ were classified as users of calcium channel blockers and $2780(81.8 \%)$ as non-users.

Main outcome measures: Partial correlations (least squares method) between rates of use of cardiovascular drugs and age standardised mortality from suicide in Swedish municipalities. Hazard ratios for risk of suicide with adjustments for difference in age and sex in users of calcium channel blockers compared with users of other hypertensive drugs. Results: Among the Swedish municipalities the use of each cardiovascular drug group except angiotensin converting enzyme inhibitors correlated significantly and positively with suicide rates. After adjustment for the use of other cardiovascular drug groups, as a substitute for the prevalence of cardiovascular morbidity, only the correlation with calcium channel
\end{abstract}

blockers remained significant $(r=0.29, \mathrm{P}<0.001)$. In the cohort study, five users and four non-users of calcium channel blockers committed suicide during the follow up until the end of 1994. The absolute risk associated with use of calcium channel blockers was 1.1 suicides per 1000 person years. The relative risk, adjusted for differences in age and sex, among users versus non-users was 5.4 (95\% confidence interval 1.4 to 20.5).

Conclusions: Use of calcium channel blockers may increase the risk of suicide.

\section{Introduction}

A recent epidemiological study reported an excess risk of depression requiring pharmacological treatment after treatment with calcium channel blockers and angiotensin converting enzyme inhibitors but not after treatment with digoxin, anti-arrhythmics, nitrates, diuretics, or $\beta$ blockers. ${ }^{1}$ There have also been case reports suggesting depression ${ }^{2-5}$ as well as psychosis ${ }^{6}$ after treatment with calcium channel blockers. As depression may promote suicide we investigated possible ecological associations between suicide rates and the rates of use of eight cardiovascular drug groups in 152 Swedish municipalities. In addition, we investigated the risk of suicide in users and non-users of calcium channel blockers who had purchased prescription drugs mainly used to treat hypertension.
Swedish Network for Pharmacoepidemiology, Foundation, Malmö University Hospital, SE-205 02 Malmö, Sweden

Gunnar Lindberg, clinical epidemiologist Jonas Ranstam, biostatistician

Arne Melander, professor

Department of Pharmaceutical Services Research, Uppsala University, Box 586, SE-751 23 Uppsala, Sweden

Kerstin Bingefors, senior lecturer

Department of Community Medicine, Lund University, Malmö University Hospital, SE-205 02 Malmö Lennart Råstam, professor

Correspondence to: Dr Lindberg gunnar.lindberg@ nepia.se

BMJ 1998;316:741-5 


\section{Methods}

This report concerns two different studies: firstly, an ecological study with data from Swedish municipalities on rates of suicide and rates of use of eight groups of cardiovascular drug groups; secondly, the hypothesis generated by this study tested in a cohort study on historical data from users of different antihypertensive drugs.

\section{The ecological study}

Sweden is administratively divided into 284 municipalities. Suicide rates for men and women standardised for age for these municipalities during the 5 year period 1989-93 were obtained from the epidemiological centre of the Swedish Board of Health and Welfare. Data on incidence of cause specific mortality were missing for eight municipalities. Data on suicide mortality (ICD-9 (international classification of diseases, 9th revision), codes E950-E959 and E980-E989) were available for 152 municipalities in which the expected number of people committing suicide during the 5 year period was more than five men and five women, as assumed from the overall suicide rates in Sweden.

Rates of use of cardiovascular drugs by outpatients, defined by the Anatomical Therapeutical Chemical (ATC) classification ${ }^{7}$ and expressed as pharmacy dispensed numbers of defined daily doses per 1000 inhabitants per year, were obtained from Apoteksbolaget (the Swedish Corporation of Pharmacies) for each of the 152 municipalities during each of the years 1989-93. The geometric means of the annual rates of use were used in the calculations.

The rates of use of eight cardiovascular drug groups-diuretics, $\beta$ blockers, calcium channel blockers, angiotensin converting enzyme inhibitors, lipid lowering agents, low dose aspirin, nitrates, and cardiac glycosides-were correlated with suicide rates by using Pearson's correlation coefficient. Partial correlation coefficients between suicide rates and rates of use of the cardiovascular drug groups were assessed to estimate a correlation coefficient for each drug group independent of variations in the rates of use of the seven other groups to use as a proxy for cardiovascular disease prevalence. All tests were two sided.

\section{The cohort study}

Data on individual prescription drug use in a municipality located in mid-eastern Sweden (population about 20000 in 1989) have been compiled and studied since the 1970s. All prescription drugs purchased by residents from pharmacies within the municipality are registered according to the ATC system. ${ }^{7}$ For the purpose of the present study all inhabitants of the municipality were identified who during 1988 or 1989 had purchased cardiovascular medications with ATC codes (in 1988 and 1989) C02 (centrally acting antiadrenergic agents, ganglion blocking antiadrenergic agents, peripherally acting antiadrenergic agents, calcium channel blockers, angiotensin converting enzyme inhibitors), C03 (diuretics), and $\mathrm{C} 07$ ( $\beta$ blockers). ATC codes for calcium channel blockers and angiotensin converting enzyme inhibitors were later changed to $\mathrm{C} 08$ and $\mathrm{C} 09$, respectively. The subjects were classified as users or non-users of calcium channel blockers. Mortality data for the cohort until the end of 1994, including the cause of death, were derived from the Swedish mortality register. ${ }^{8}$ Deaths from suicide were defined by the ICD-9 codes E950-E959 and E980-E989. The codes E980-E989 include cases with uncertain intention for suicide. Data on purchased medications and cause of death were linked by the Swedish personal identification number.

Differences in risk of suicide were evaluated by the Kaplan-Meier method and the log rank test. Multivariate adjustments for differences in age and sex were performed with the proportional hazards method. All $\mathrm{P}$ values are two sided.

\section{Results}

The ecological study

The number of suicides in the 152 municipalities during the 5 year period ranged from 5 to 652 . The total number of suicides in the municipalities during this period was 5648. The total population was 7.3 million, and the mean (range) municipality population was 48042 (13 722 - 679 364) in 1991. Age adjusted suicide rates varied from 0.76 to 3.69 deaths per 10000 inhabitants per year. The mean (SD) suicide rate for the 152 municipalities was $2.06(0.49)$ suicides per 10000 inhabitants per year. The rates of use were 79.7 defined daily doses per 1000 inhabitants per day for diuretics, 36.8 for $\beta$ blockers, 22.5 for calcium channel blockers, 20.4 for nitrates, 16.0 for angiotensin converting enzyme inhibitors, 15.3 for cardiac glycosides, 9.4 for low dose aspirin, and 2.9 for lipid lowering agents.

The correlation coefficients for the relations between rates of drug use and rates of suicide are given in table 1. Except for angiotensin converting enzyme

Table 1 Correlation coefficients for rates of use of cardiovascular drug groups and rates of suicide in 152 Swedish municipalities, 1989-93

\begin{tabular}{llc} 
Drug group & Unadjusted & Adjusted† \\
\hline Diuretics & $0.27^{\star \star}$ & 0.14 \\
\hline$\beta$ blockers & $0.20^{\star}$ & -0.11 \\
\hline Calcium channel blockers & $0.36^{\star \star \star}$ & $0.29^{\star \star \star}$ \\
\hline ACE inhibitors & 0.11 & -0.09 \\
\hline Lipid lowering agents & $0.19^{\star *}$ & 0.16 \\
\hline Low dose aspirin & $0.18^{\star}$ & -0.03 \\
\hline Nitrates & $0.17^{\star}$ & -0.12 \\
\hline Cardiac glycosides & $0.20^{\star}$ & -0.06 \\
\hline
\end{tabular}

ACE=Angiotensin converting enzyme inhibitors.

†Adjusted for differences in rates of use of all other cardiovascular agents. ${ }^{*} \mathrm{P}<0.05 ;{ }^{*} \mathrm{P}<0.01 ;{ }^{* *} \mathrm{P}<0.001$

Table 2 Correlation coefficients for rates of use of calcium channel blockers and rates of suicide in 152 Swedish municipalities, 1989-93

\begin{tabular}{lll} 
Drug & Unadjusted & Adjusted† \\
\hline All vasoselective drugs & $0.31^{\star \star \star}$ & $0.21^{\star}$ \\
\hline Felodipine & $0.24^{\star *}$ & 0.14 \\
\hline Nifedipine & $0.21^{\star \star}$ & 0.12 \\
\hline All cardioselective drugs & $0.39^{\star \star *}$ & $0.17^{\star}$ \\
\hline Verapamil & $0.22^{\star \star}$ & 0.08 \\
\hline Diltiazem & $0.28^{\star *}$ & $0.19^{\star}$ \\
\hline
\end{tabular}

tAdjusted for differences in rates of use of all agents included in table 1 except calcium channel blockers.

${ }^{\star} \mathrm{P}<0.05 ;{ }^{*} \mathrm{P}<0.01 ;{ }^{*}{ }^{*} \mathrm{P}<0.001$. 


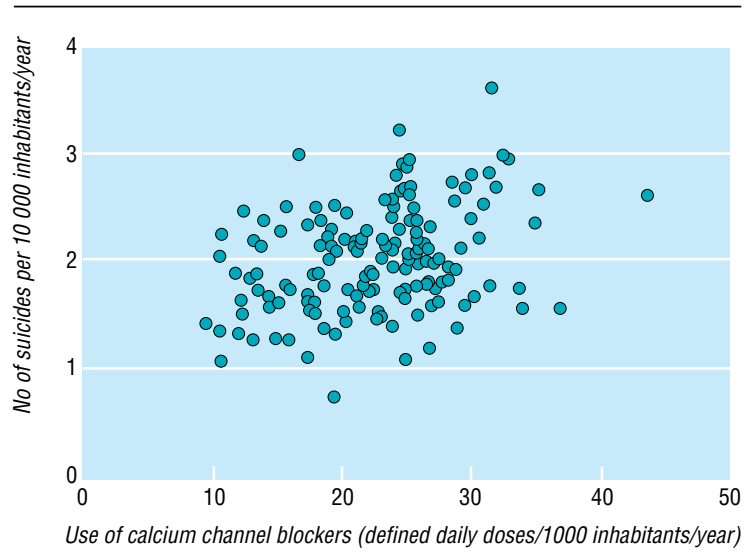

Fig 1 Correlation between rates of use of calcium channel blockers and rates of suicide in 152 Swedish municipalities

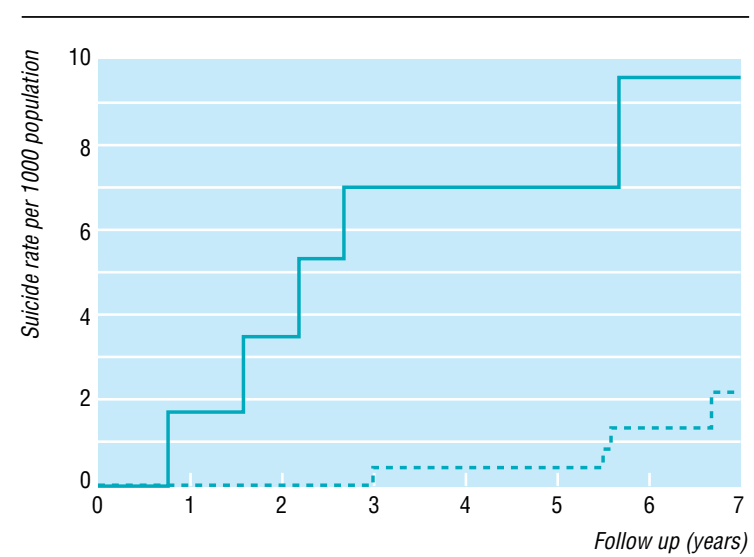

Fig 2 Cumulative rate of suicide over 7 years' follow up in 617 users (continuous line) and 2780 non-users (dotted line) of calcium channel blockers

inhibitors, the rates of use correlated significantly with the suicide rates. The highest correlation coefficient was seen for calcium channel blockers $(r=0.36$, $\mathrm{P}<0.001$ ) (figure 1). After adjustment for differences in the rates of use of the other drug groups, only the rates of use of calcium channel blockers correlated significantly with the suicide rates $(r=0.29, \mathrm{P}<0.001)$ (table 1). Additional adjustment for the proportion of men living in the municipality did not alter this result.

The rates of use of two predominantly cardioselective and two predominantly vasoselective calcium channel blockers also correlated significantly with suicide rates (table 2). After adjustment for the rates of use of the seven other types of cardiovascular drug groups, the use of both dihydropyridine and benzothiazepine derivatives remained significantly correlated with suicide rates. When we adjusted for differences in use of the seven other cardiovascular drug groups, the correlation coefficient was 0.21 for dihydropyridine derivates and 0.18 for benzothiazepine derivatives. The rates of use of diltiazem had the closest correlation with suicide rates (table 2).

\section{The cohort study}

In all, 3397 patients were identified as purchasers of drugs with ATC codes C02, C03, and C07 in 1988 and 1989. Of them, 617 (18.2\%) were classified as users of calcium channel blockers (nifedipine, verapamil, diltiazem, and felodipine) and $2780(81.8 \%)$ as non-users. During the follow up, from the date of purchase until the end of 1994, five users of calcium channel blockers (three men and two women, one with uncertain intent) and four non-users (three men and one woman, none with uncertain intent) committed suicide. The 7 year suicide risks were 9.7 and 2.2 per 1000 persons for calcium channel blocker users and non-users, respectively. The difference in suicide risk was significant $(\mathrm{P}=0.002$. $)$ The average annual absolute risk associated with use of calcium channel blockers was therefore 1.1 suicides per 1000 persons. After adjustment for differences in age and sex the relative risk among users versus nonusers was 5.4 (95\% confidence interval 1.4 to 20.5). Figure 2 illustrates the cumulative suicide rates during follow up of users and non-users of calcium channel blockers.

\section{Discussion}

In the past several groups of cardiovascular drug have been associated with depressive disorders. As suicide is a serious consequence of depression, the current studies were undertaken to evaluate the possible influence of widely used drugs on risk of suicide. The correlation between use of calcium channel blockers and suicide rates found in the ecological study led us to design a cohort study to test if, among users of antihypertensive drugs, subjects using calcium channel blockers had a higher risk of suicide than subjects not using calcium channel blockers.

Diltiazem, ${ }^{2}$ nifedipine, ${ }^{3}$ and verapamil ${ }^{4}$ have been associated with depressive disorders in case reports and also in a previous epidemiological study that used data on individual prescriptions of calcium channel blockers and antidepressants. ${ }^{1}$ The two current studies imply that calcium channel blockers may also promote suicide. In the ecological study the estimated correlation suggests that about one tenth of the intermunicipality variation in risk of suicide is related to the use of calcium channel blockers. In the cohort study the suicide risk in users of calcium channel blockers adjusted for sex and age was fivefold compared with the risk in non-users treated with other antihypertensive agents.

Clinical trials have a limited ability to detect infrequent or late adverse effects or adverse effects resulting in common symptoms. They also often use a number of inclusion and exclusion criteria, thus reducing their generalisability. Studies with a long follow up and studies encompassing the whole population are therefore needed.

In contrast with most clinical trials the current cohort study included all identified users of the study drugs. Consequently the generalisability of the study is high. In the ecological study low populated municipalities with few expected suicides were excluded, so the influence from extreme rates in combination with small number of events was avoided.

In Sweden, as in most other countries, men have a higher incidence of suicide than women. Men are also more likely to be prescribed a calcium channel blocker (unpublished data on file). In our cohort study the estimated rates of suicide were adjusted for differences in age and sex, and, in the ecological study, the suicide rates were adjusted for differences in age. Additional 
adjustment of the ecological correlations for the proportion of men in the municipalities did not affect the results (no data given), thus we can eliminate age or sex differences as confounders.

Comorbidity such as cardiovascular diseases might have promoted depressive disorders and suicide. In the ecological study this problem was dealt with by adjusting the correlation for each tested cardiovascular drug group with the rate of use of the seven other drug groups. In the cohort study all subjects were treated with antihypertensive drugs. It follows that although no detailed clinical data were available, users and non-users of calcium channel blockers most probably had similar medical backgrounds.

Populations with a high prevalence of cardiovascular diseases also have a high suicide risk. In the ecological study the rates of use of all but one evaluated cardiovascular drug group also correlated significantly with the suicide rates before adjustment for the rates of use of the other drug groups. After adjustment, however, only the rate of use of calcium channel blockers was significantly and positively correlated with suicide rates (see table 1). Accordingly, the increased suicide risk linked to use of calcium channel blockers would seem independent of cardiovascular comorbidity.

\section{Links with depression}

Calcium channel blockers are often prescribed to treat angina pectoris. If angina pectoris causes depression, this might form a link to suicidal behaviour. Nitrates are also prescribed to treat angina pectoris, however, and the rates of use of nitrates did not correlate with suicide rates when we adjusted for the rates of use of other cardiovascular drug groups. Neither did the use of angiotensin converting enzyme inhibitors, often prescribed to patients with diabetes, correlate with suicide rates. Thus, it seems unlikely that the presence of angina pectoris or diabetes help to explain the linking of calcium channel blockers to depression and increased suicide risk.

Other reasons to prescribe calcium channel blockers might have been greater difficulty in achieving control of blood pressure or adverse effects from other antihypertensive drugs. Again, it is not very likely that such circumstances have enough penetrative power to link calcium channel blockers to depression and suicide risk.

In the late 1980s $\beta$ blockers were suspected of inducing depression. ${ }^{9}$ Therefore, other antihypertensive or antiangina drugs might have been chosen for patients with depression. If calcium channel blockers were used particularly often in depressed patients, an increased suicide risk would appear in users of calcium channel blockers even though the drugs per se would not promote depression and suicide. To behave as a confounder, however, the confounding variable has to be substantially correlated to the tested exposition as well as to the outcome. Therefore, to achieve a high increase in suicide risk by confounding from selective prescribing of calcium channel blockers to depressed patients, most depressed patients with increased risk of suicide and few non-depressed patients should have been prescribed calcium channel blockers. Only about half of depressed patients are correctly diagnosed by their primary care physicians, ${ }^{10}$ however, and use of
Key messages

- Clinical trials have a limited ability to detect infrequent adverse effects, so postmarketing reports on adverse effects and observational epidemiological studies are necessary

- The present investigations, including one cross sectional ecological study and one population based cohort study, suggest an increased risk of suicide in users of calcium channel blockers

- The results are in accordance with a depressive effect of calcium channel blockers suggested by case reports and a recent epidemiological study

- Channel blockers should be considered a possible cause of depression and suicide

calcium channel blockers is also obviously common in patients without depression.

In the cohort study population patients were classified as users and non-users of calcium channel blockers at the time of inclusion. Some of the former might have stopped taking calcium channel blockers shortly after inclusion and some non-users might have been prescribed calcium channel blockers after inclusion. Moreover, both studies take drugs purchased from pharmacies into account. It is not certain that each purchased drug was used, and some inhabitants may not have purchased drugs from pharmacies in their home municipality. Provided that the misclassification was independent of the outcome, however, misclassification of exposure would probably have resulted in underestimation of the true association.

One way to interpret the results of the ecological study is to assume that most, if not all, cardiovascular agents have some depressive effect, calcium channel blockers being most prominent. As the studied cardiovascular agents were very heterogeneous, however, a common effect on mood is unlikely.

In contrast with most other cardiovascular agents, calcium channel blockers influence secretory and contractile mechanisms in many different types of cells. Because of their lipophilic properties they easily penetrate the blood-brain barrier. Hence they have access to and may interfere with neurones and receptors involved in the regulation of mood. As calcium channel blockers differ in selectivity and in kinetics their influence on the central nervous system may vary. In the ecological study, however, the rates of use of both dihydropyridine and benzothiazepine derivatives correlated significantly and those of phenylalkylamine derivatives non-significantly with suicide rates after adjustment for the rates of use of other cardiovascular drug groups. Hence it seems likely that calcium channel blockade per se is involved in the increased risk of suicide. A calcium channel effect of dihydropyridines in affective disorders has also been suggested previously. ${ }^{5}$

In conclusion, use of calcium channel blockers may increase risk of suicide. A depressive effect of these drugs has been suggested and may constitute a link with risk of suicide. The consequences of treatment with calcium channel blockers should be further inves- 
tigated with respect to depressive disorders and suicide. Calcium channel blockers should be considered as a possible cause of depression and suicide

Contributors: GL initiated the ecological and cohort studies, formulated the study aims, designed the layouts, organised the data collection, and participated in the statistical analysis and interpretation of results and in writing the paper. KB participated in collecting and interpreting results for the cohort study. JR participated in the statistical analysis and interpretation of the data and contributed to writing the paper. LR participated in the interpretation of the data and the writing of the paper. AM, head of the Swedish Network for Pharmacoepidemiology (NEPI), participated in the interpretation of the data and contributed to writing and editing the paper.

Funding: The NEPI Foundation (the Swedish Network of Pharmacoepidemiology) and the Swedish National Institute of Public Health.

Conflict of interest: None.

1 Hallas J. Evidence of depression provoked by cardiovascular medication: a prescription sequence symmetry analysis. Epidemiology 1996;7:478-84.
Biriell C, McEwen J, Sanz E. Depression associated with diltiazem. BMJ 1989;299:796.

3 Hullett FJ, Potkin SG, Levy AB, Ciasca R. Depression associated with nifedipine-induced calcium channel blockade. Am $J$ Psychiatry $1988 ; 145: 1277-9$.

4 Dassylva B. Verapamil may cause depression. Can J Psychiatry 1993:38:299-300.

5 McAllister-Williams RH. Calcium-channel blockade and depressive illness. Br J Psychiatry 1990;157:618-9.

6 Kahn JK. Nifedipine-associated acute psychosis. Am J Med 1986;81:705-6.

7 Capellà D. Descriptive tools and analysis. In: Dukes MNG, ed. Drug utilisation studies: methods and uses. Copenhagen: WHO Regional Office for Europe, 1993:55-78. (WHO regional publication. European series: No 45.)

8 Bolander AM. Mortality statistics in Sweden and its neighbouring countries. Advantages and hazards inherent in systems and materials. In: Boström H, Lungstedt N, eds. Medical aspects of mortality statistics. Stockholm: Almquist and Wiksell, 1981:236-55.

9 Avorn J, Everitt DE, Weiss S. Increased antidepressant use in patients prescribed beta-blockers. JAMA 1986;255:357-60.

10 Nielsen AC, Williams TA. Depression in ambulatory medical patients. Prevalence by self-reported questionnaire and recognition by nonpsychiatric physicians. Arch Gen Psychiatry 1980;37:999-1004.

(Accepted 11 November 1997)

\title{
QT and QTc dispersion are accurate predictors of cardiac death in newly diagnosed non-insulin dependent diabetes: cohort study
}

\author{
Abdul A O Naas, Neil C Davidson, Chris Thompson, Fraser Cummings, Simon A Ogston, \\ Roland T Jung, Ray W Newton, Allan D Struthers
}

Patients with non-insulin dependent diabetes mellitus have an excess risk of dying from cardiovascular disease. One small study suggested that a prolonged QT interval could predict cardiac death in patients with diabetic nephropathy who have received insulin treatment. The question now is whether the same is true in newly diagnosed diabetes in patients who have no apparent complications. In addition, QT dispersion, a new but related electrocardiographic variable, predicts cardiac death in patients who have chronic heart failure, peripheral vascular disease, or essential hypertension. ${ }^{1-3}$ We investigated whether it also predicted cardiac death in diabetic patients.

\section{Subjects, methods, and results}

The study group of 182 patients with non-insulin dependent diabetes mellitus (103 men; mean age 52.8 (SD 8.5) years) represented the Dundee cohort of the United Kingdom prospective diabetes study, which was recruited between 1982 and 1988. Patients were followed up for a mean of 10.3 (1.7) years. The inclusion and exclusion criteria of the study have been reported elsewhere. Patients with overt cardiac disease at baseline were excluded. A single observer (AAON) measured QT intervals as described previously. ${ }^{1-3}$ Cardiac death was mostly classified at the coordinating centre in Oxford, using the codes of the international classification of diseases, ninth revision. All analysis was done by Cox regression analysis, with cardiac death as the sole end point. We used forward stepwise analysis, each time using all three QT variables along with age, systolic blood pressure, sex, smoking, blood glucose concentration, and antihypertensive drug. As a result, we identified age, systolic blood pressure, sex, diuretics, and all QT variables as the potentially important variables. Finally we fitted the regression using these four variables with each of the three QT variables.

In those who had a cardiac death, the mean time of death after the baseline electrocardiogram was 7.3 (3.2) years; after the 3 year electrocardiogram it was 4.9 (2.3) years and after the 6 year electrocardiogram 3.8 (1.0) years. The table shows that QTc max, QTc dispersion, and QT dispersion are all highly significant and independent predictors of cardiac death at baseline, at 3 years, and at 6 years. In multivariate analysis they outperformed all other predictors.

\section{Comment}

Our main finding was that QT dispersion, QTc dispersion, and QTc max are excellent predictors of cardiac death in patients with non-insulin dependent diabetes mellitus. QTc interval analysis has two major advantages over other possible ways of stratifying risk in patients. Firstly, measurements of QTc interval are easily obtained with a non-invasive routine test: other potential predictors of cardiac death often require extra testing with specialised equipment. Secondly, comparisons between QTc dispersion and microalbuminuria suggest that QTc dispersion is a better predictor of cardiac death. ${ }^{4}$ A QTc dispersion $>78 \mathrm{~ms}$ at year 6 in this study had $100 \%$ sensitivity and $90 \%$ specificity, giving an odds ratio of 9.0 , whereas the odds
Department of Clinical Pharmacology and Therapeutics, Ninewells Hospital and Medical School, Dundee DD1 9SY Abdul A O Naas, clinical research fellow Neil C Davidson, British Heart Foundation research fellow

Allan D Struthers, consultant physician

Diabetes Centre, Ninewells Hospital and Medical School Chris Thompson, senior registrar Fraser Cummings, medical student Roland T Jung, consultant physician Ray W Newton, consultant physician

Department of Epidemiology, Ninewells Hospital and Medical School Simon A Ogston, senior lecturer

Correspondence to: Professor Struthers

BMJ 1998;316:745-6 